\title{
Graphene-Integrated Metamaterial Device for All-Electrical Polarization Control of Terahertz Quantum Cascade Lasers
}

Stephen J. Kindness, ${ }^{* \dagger}{ }^{\dagger}$ Nikita W. Almond, ${ }^{\dagger}$ Wladislaw Michailow, $^{\dagger}$ Binbin Wei, $^{\dagger}$ Lukas A. Jakob, $^{\dagger}$ Kaveh Delfanazari, ${ }^{\dagger, \dagger}$ Philipp Braeuninger-Weimer, ${ }^{\ddagger}{ }^{\ddagger}$ Stephan Hofmann, ${ }^{\ddagger}$ Harvey E. Beere, ${ }^{\dagger}$ David A. Ritchie, ${ }^{\dagger}$ and Riccardo Degl'Innocenti ${ }^{\S}{ }^{\circ}$

${ }^{\dagger}$ Cavendish Laboratory, University of Cambridge, J J Thomson Avenue, Cambridge CB3 OHE, U.K.

"Department of Engineering, University of Cambridge, $9 \mathrm{~J} \mathrm{~J}$ Thomson Avenue, Cambridge CB3 OFA, U.K.

${ }^{\S}$ Department of Engineering, University of Lancaster, Bailrigg, Lancaster LA1 4YW, U.K.

\author{
Supporting Information
}

ABSTRACT: Optoelectronic modulators that operate by the electrical tuning of plasmonic resonator structures have demonstrated fast $(>\mathrm{MHz})$ manipulation of terahertz $(\mathrm{THz})$ radiation for communications, imaging, and spectroscopy applications. Among this class of $\mathrm{THz}$ device, chiral metamaterial-based polarization modulators have attracted increasing attention due to the importance of $\mathrm{THz}$ polarization control for chemistry, biology, and spectroscopy applications, as well as for $\mathrm{THz}$ communication protocols. In this paper, active polarization modulation of a $\mathrm{THz}$ quantum cascade laser is demonstrated by the electrical tuning of a $2 \mathrm{D}$ chiral metamaterial array. The operating principle of this device is based on

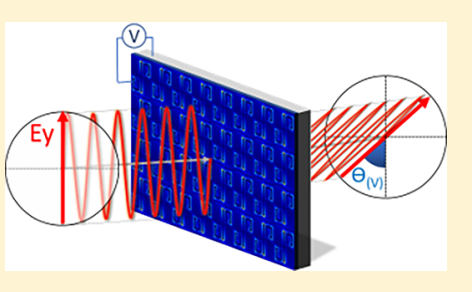
an electromagnetically induced transparency analogue, produced by the coupling between a bright resonator and two dark resonators. The orientation of these resonators is such that a radiating electric dipole orthogonal to the incident electric field polarization is induced, causing a rotation of the polarization angle of the transmitted radiation. By variably dampening the dark resonators using graphene, the coupling condition is electrically modulated such that continuous tuning of the transmitted polarization angle is achieved. This device, operating at room temperature, can be readily implemented as a fast, optoelectronic, polarization modulator with a maximum tuning range of 20 degrees at $1.75 \mathrm{THz}$, with demonstrated reconfiguration speeds of $>5 \mathrm{MHz}$.

KEYWORDS: terahertz, graphene, chiral metamaterials, coupled plasmonic resonators, optoelectronics, polarization modulation, quantum cascade laser

$\mathrm{T}$ he motivation for building electrically controllable polarization devices lies in the myriad of applications where active control of $\mathrm{THz}$ radiation polarization is essential. $\mathrm{THz}$ communications is an emerging application, with protocols such as polarization shift keying ${ }^{1}$ and polarization division multiplexing ${ }^{2}$ expected to be important parts of this technology where speed of modulation is paramount. A device that could actively control the polarization condition of $\mathrm{THz}$ radiation would be an important tool in several fields of research. This would address fundamental challenges in material characterization, for instance, characterization of topological insulators, ${ }^{3,4}$ as well as probing the birefringence of materials, ${ }^{5}$ which has applications, for example, in the pharmaceutical industry. ${ }^{6,7}$ Finally, an active control of $\mathrm{THz}$ light polarization enables the probing and characterization of inherently chiral biomolecules, such as DNA, RNA, and proteins. ${ }^{8,9}$ Despite the breadth and importance of all the aforementioned applications, there is a distinct shortage of available modular devices capable of controlling $\mathrm{THz}$ polarization electrically.

Various $\mathrm{THz}$ sources with variable output polarization conditions have been demonstrated; ${ }^{10,11}$ however these approaches have integrated the $\mathrm{THz}$ source with the polarization element. A more versatile approach would be to design a polarization modulator device that can be integrated with any $\mathrm{THz}$ source such as a standard quantum cascade laser (QCL), which is a narrow-bandwidth, powerful, and ultrastable THz source. ${ }^{12}$ In order to modify the polarization state of the QCL-emitted radiation, which is transverse-magnetic linearly polarized because of the intersubband transition rules, broadband grating ${ }^{13-16}$ based devices could be used; however, chiral metamaterial arrays represent an optimal solution, as they display dramatic frequency-dependent characteristics such as optical activity and circular dichroism.

There is a wealth of static chiral metamaterial structures that have been shown to possess these properties; ${ }^{17-22}$ however, realizing actively tunable chiral metamaterial devices is fundamentally more complex. Several approaches have been reported. For example, Kan et al. $^{23}$ demonstrated effective polarization rotation by using microelectromechanical systems (MEMS). Alternatively, the introduction of photoactive materials, such as silicon, proved to be an effective method in combination with chiral complex structures ${ }^{24}$ to induce

Received: March 15, 2019

Published: May 22, 2019 
polarization modulation. Single-layer chiral metamaterial devices, ${ }^{25}$ as well as a double-layer devices, ${ }^{26}$ have incorporated photoactive silicon to actively tune the polarization angle and handedness, respectively.

The aforementioned modulation approaches proved to be effective, although MEMS devices suffer from intrinsically low maximum modulation speeds $(100 \mathrm{kHz})$, and photoactive schemes require complex optical systems. Graphene instead represents an ideal tunable material, as its conductivity can be modulated via electrostatic backgating, ${ }^{27}$ with carrier concentrations of $10^{14} \mathrm{~cm}^{-2}$ achievable, ${ }^{28,29}$ and has been successfully integrated with metamaterial structures to produce a range of modulators working in the $\mathrm{THz}$ and infrared. ${ }^{30-32}$ These devices have shown to be effective when integrated with $\mathrm{THz}$ QCLs, ${ }^{33,34}$ providing $100 \%$ amplitude modulation depths, ${ }^{35}$ as well as modulation speeds of $>100 \mathrm{MHz} .{ }^{36}$ This paper will look to integrate a single layer, chiral metamaterial array with graphene to realize an electrically tunable polarization modulator that demonstrates polarization control of a $\mathrm{THz}$ QCL.

\section{RESULTS AND DISCUSSION}

Outline. Active polarization modulation was achieved by integrating a single-layer chiral metamaterial structure with electrostatically gated graphene to variably dampen the resonance condition and hence modify the transmitted polarization through the device. This device makes use of a coupled bright and dark resonator structure ${ }^{37-41}$ that has a built-in $2 \mathrm{D}$ chirality $^{22}$ with the device exhibiting electrically tunable optical activity. As a consequence, the polarization angle of transmitted radiation through the device can be actively controlled. A graphene tuning mechanism similar to the approach in ref 44 was used, which involves variably dampening one of the coupled resonators with graphene. An illustration of the metamaterial structure is shown in Figure 1, with the central dipole resonator acting as a bright resonator when excited with incident radiation polarized in the $y$ direction $\left(E_{\mathrm{y}}\right)$. The two $\mathrm{C}$-shaped resonators on either side represent dark resonators, which are not excited directly by the incident radiation. These dark resonators are indirectly excited from capacitive coupling with the central bright resonator, resulting in resonating electric dipoles in the $x$ direction, $P_{x}$ illustrated in Figure 1(a). These resonating $P_{x}$ dipoles re-emit $\mathrm{THz}$ radiation which is plane polarized in the $x$ direction $\left(E_{x}\right)$, causing a rotation effect on the polarization of the transmitted radiation. This effect can be modulated by tuning the graphene conductivity via electrostatic backgating, using the thin metal lines to electrically contact the graphene. This results in a transmitted polarization angle that is dependent on the applied backgate voltage across the device.

Device Fabrication. The device was fabricated on a boron p-doped silicon substrate with a $300 \mathrm{~nm}$ insulating layer of $\mathrm{SiO}_{2}$ to realize the electrostatic backgate. The total metamaterial area was $1.7 \mathrm{~mm} \times 1.7 \mathrm{~mm}$, containing $27 \times$ 27 individual unit cells. Standard photolithography techniques were first used to define the bond pads, followed by the deposition of $10 / 100 \mathrm{~nm}$ of $\mathrm{Ti} / \mathrm{Au}$ via thermal evaporation. The coupled metal resonator arrays were then fabricated using electron beam (e-beam) lithography, thermal evaporation of $10 / 70 \mathrm{~nm}$ of $\mathrm{Ti} / \mathrm{Au}$, and liftoff. The specific lithographic tuning parameters are shown in SI.1 (Supporting Information). Graphene was grown via chemical vapor deposition (CVD) on a Cu catalyst ${ }^{42}$ and subsequently transferred on top of the gold
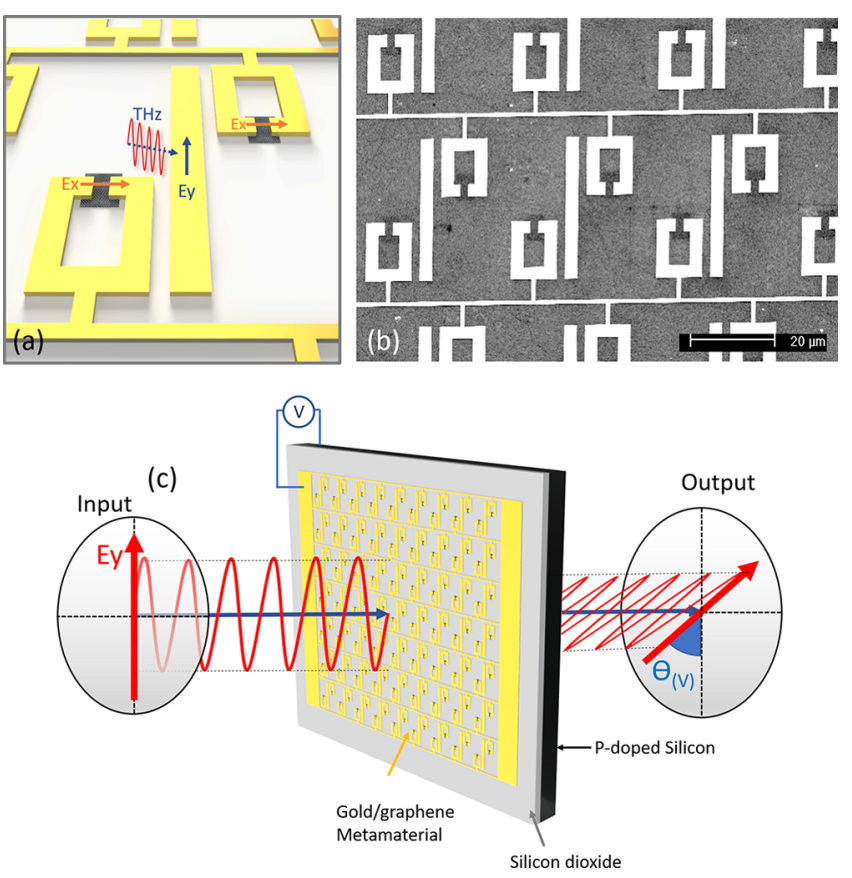

Figure 1. (a) Diagram of device unit cell showing incident radiation polarized in the $E_{y}$ direction. (b) SEM image showing a section of the device array. Graphene patches are seen in the capacitive gap of the dark resonators. (c) Illustration of working principle of polarization rotation device.

resonator arrays using a sacrificial polymer layer. ${ }^{43}$ It was then patterned into $6 \mu \mathrm{m} \times 4 \mu \mathrm{m}$ patches using e-beam lithography with oxygen plasma etching. A scanning electron microscope (SEM) image of the fabricated device surface is shown in Figure 1(b). The sample was mounted and wire-bonded for electrical biasing on a chip carrier configured for transmission measurements, with silver paste electrically contacting the pdoped silicon substrate for backgate biasing purposes. A 1.2 $\mathrm{mm} \times 1 \mathrm{~mm}$ continuous, uniform graphene area was included next to the device array on the same substrate for the electrical characterization of the graphene conductivity as a function of the backgate voltage. The same conductivity range measured from the uniform area was assigned to the graphene patches in the metamaterial devices, since the graphene growth and transfer, as well as the processing, had been carried out at the same time. A diagram of the full device illustrating the backgating method is shown in Figure 1(c). The working principle of the device is also illustrated in this figure, with the device displaying an electrically controllable optical activity. When incident $E_{y}$ radiation passes through the device, the polarization angle, $\theta(V)$, of the transmitted radiation is rotated by an angle that is dependent on the applied backgate voltage.

Simulation: Finite Element Method. A set of simulations were performed with the finite element method (FEM) software COMSOL Multiphysics V 5.3a, in order to gain insight into the polarization rotation phenomenon and to inform optimal device parameters. A base unit cell was defined, as shown in Figure 2, with Floquet boundary conditions used to simulate a continuous metamaterial array. The incoming $\mathrm{THz}$ radiation was simulated with a top port emitting $E_{y^{-}}$ polarized plane waves toward the device structure. The Drude model was employed to describe the complex conductivity of both graphene and gold; however, for simplicity, only the DC sheet conductivity for graphene was quoted in the following 


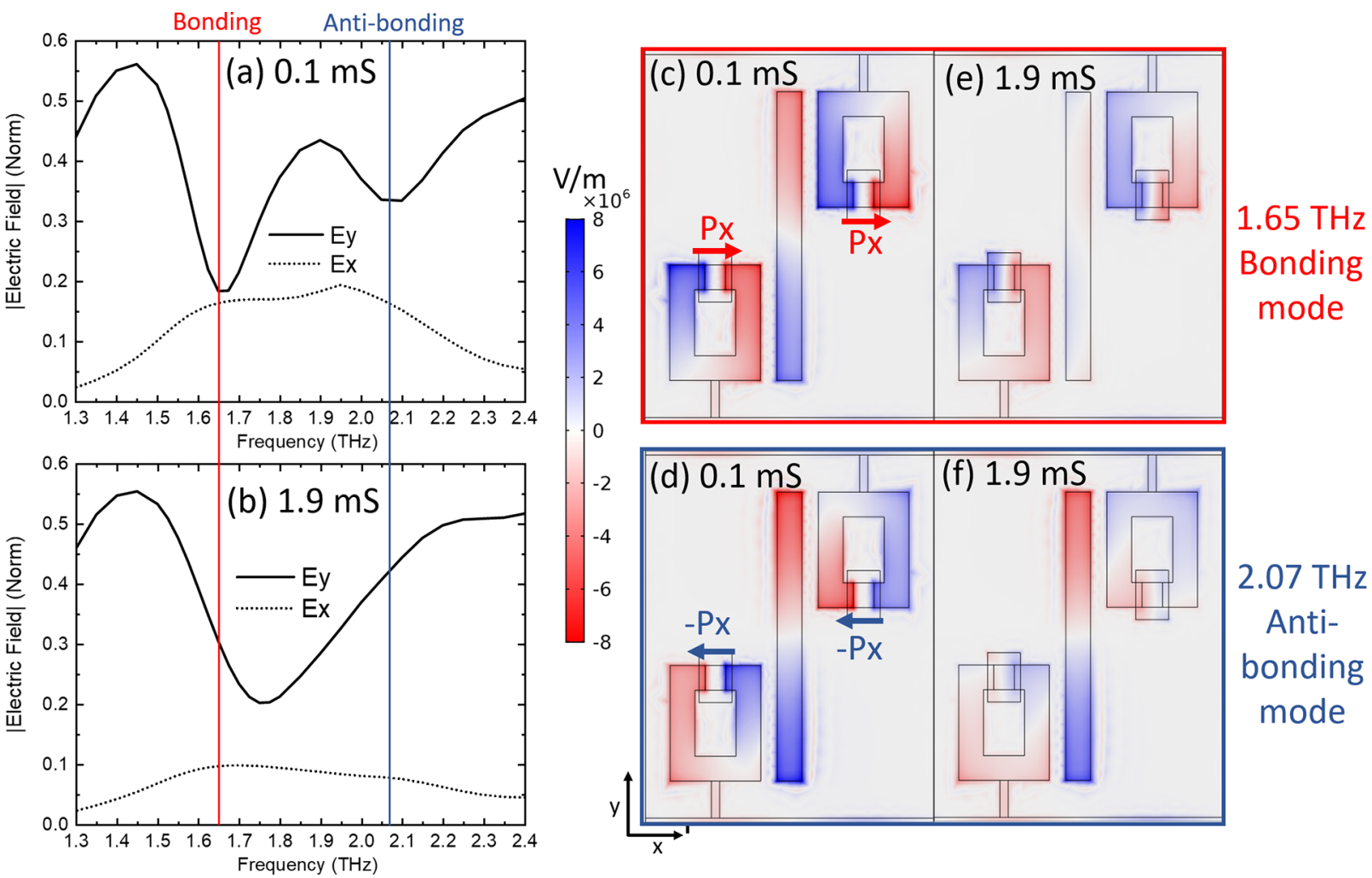

Figure 2. Comsol simulation of transmitted $\left|E_{y}\right|$ and reradiated $\left|E_{x}\right|$ components normalized to incident $E_{y}$ intensity for graphene sheet conductivities of (a) $0.1 \mathrm{mS}$ and (b) $1.9 \mathrm{mS}$. (c-f) E-field intensity in the $z$ direction simulated at a distance of $20 \mathrm{~nm}$ from the metal surface produced by incident plane wave excitation of $E_{y}$-polarized radiation with a nominal power of $1 \mathrm{~W}$ across the unit cell. (c and e) Indication of carrier concentration due to excitation of bonding mode with incident radiation at $1.65 \mathrm{THz}$ for graphene conductivities of 0.1 and $1.9 \mathrm{mS}$, respectively. ( $\mathrm{d}$ and $\mathrm{f}$ ) Indication of carrier concentration due to excitation of the antibonding mode with incident radiation at $2.07 \mathrm{THz}$ for graphene conductivities of 0.1 and $1.9 \mathrm{mS}$, respectively.

figures when describing different graphene conductivities. The specific Drude parameters are reported in more detail in Section SI.2.

The polarization plane angle of transmitted radiation depends on the relative weight of the $E_{x}$ component reemitted by the C-shaped resonators, and the transmitted $E_{y}$ component, which is not reflected by the central dipole resonator. Figure 2(a) and (b) show these simulated components as a function of frequency for graphene DC sheet conductivity values of 0.1 and $1.9 \mathrm{mS}$, respectively. When the graphene sheet conductivity is $0.1 \mathrm{mS}$, the dampening of the dark resonators is low; hence the metamaterial is in a coupled resonator regime, with a bonding resonance and antibonding resonance visible at 1.65 and $2.07 \mathrm{THz}$, respectively, which are identified by dips in $\left|E_{y}\right|$ shown in Figure 2(a). Figure 2(b) shows the response of the metamaterial when the graphene sheet conductivity is increased to $1.9 \mathrm{mS}$, with only one resonance visible at 1.75 $\mathrm{THz}$ and with a clear overall reduction in $\left|E_{x}\right|$. In this case the dampening of the dark resonators is much greater due to the increased graphene conductivity, effectively switching the dark resonators off and converting the metamaterial from a coupled resonator regime to a single dipole resonator regime.

The peak charge carrier concentration and polarity on the surface of the metal resonators are probed by simulating the electric field in the $z$ direction at a height of $20 \mathrm{~nm}$ above the metal surface when excited by incident $E_{y}$-polarized radiation. Excitation frequencies of 1.65 and $2.07 \mathrm{THz}$ are investigated for graphene sheet conductivities of 0.1 and $1.9 \mathrm{mS}$ with the four different scenarios simulated in Figure 2(c)-(f). The carrier concentrations excited at the bonding and antibonding resonances are shown in Figure 2(c) and (d), respectively, both simulations using a graphene sheet conductivity of 0.1 $\mathrm{mS}$. In Figure 2(c), the two C-shaped resonators have dipole moments pointing in the $x$ direction, causing $E_{x}$-polarized radiation to be emitted. A similar $P_{x}$ dipole is observed for the antibonding resonance shown in Figure 2(d); however the polarity of the dipole has flipped. As the graphene conductivity increases, the localized charge carrier concentration in the central dipole resonator and C-shaped resonators decreases, with the high graphene dampening switching the resonators from a coupled regime to a single-resonator regime, shown in Figure 2(e) and (f). As a result of the weaker charge carrier concentration, a larger proportion of the incident $E_{y}$ radiation passes through the device without being reflected, and less $E_{x}$ power is re-emitted by radiating dipoles in the $\mathrm{C}$-shaped resonators. Therefore, as the graphene conductivity increases, the polarization angle will be rotated back toward the incident $E_{y}$ plane polarization, resulting in a continuous tuning of the transmitted polarization angle.

Simulation: Equivalent Circuit Model. An LCR equivalent electric circuit model was developed to further understand the working principle of the metamaterial design building on similar reported models. ${ }^{44}$ The individual resonators in isolation are modeled as antennas that have a frequency-dependent impedance described by a standard LCR electrical circuit. The coupling between the resonators was taken into account using parallel coupling capacitors between 
the bright resonator circuit and the two dark resonator circuits, as shown in Figure 3. The quantitative values for the circuit
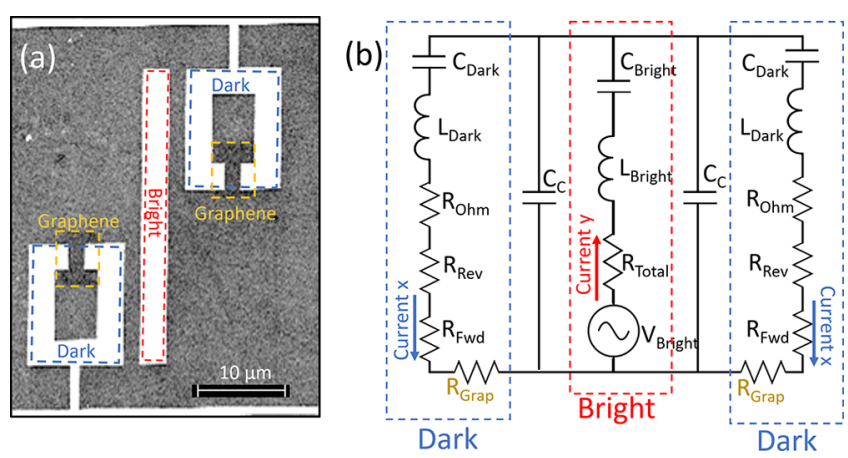

Figure 3. (a) SEM image of a single unit cell of the metamaterial design. (b) Equivalent circuit model for the device.

model parameters are derived from the FEM Comsol simulations and discussed in more detail in Section SI.3. The AC voltage source labeled $V_{\text {Bright }}$ describes the electromotive force arising from the incident $\mathrm{THz}$ radiation in the central dipole resonator. The $\mathrm{C}$-shaped resonator circuits on either side have no driving voltage source associated with them, as they are acting as dark resonators. The capacitors take into account the energy stored in the electric field due to the buildup of charges in the resonators, and the inductors reflect the magnetic energy stored from the induced current in each resonator. $R_{\text {Total }}$ describes the combined energy lost due to ohmic losses and radiation losses in the bright resonator. The loss in the dark resonators are divided into their constituent parts with the ohmic losses, labeled $R_{\mathrm{Ohm}}$, and the radiative losses in the reverse and forward direction, $R_{\mathrm{Rev}}$ and $R_{\mathrm{Fwd}}$, respectively. To account for the power lost due to dissipation in graphene, a variable resistor in series, $R_{\text {Grap }}$, was included. For simplicity, due to the low scattering time of the graphene, only the real part of the conductivity was considered. The graphene patch dissipates power and hence dampens the dark resonator according to the following equation: ${ }^{45}$

$$
P_{\text {Diss }}=\sigma_{\text {Graph }}\left\|E_{\text {Gap }}\right\|^{2}
$$

The power dissipated, $P_{\text {Diss }}$, in the graphene is dependent on the time-averaged electric field magnitude across the capacitive gap of the dark resonators, $\left\|E_{\mathrm{Gap}}\right\|$, and the real conductivity of the graphene patch, $\sigma_{\mathrm{Graph}}$. To determine the transmitted $E_{y}$ power through the device, $T$, the power drawn from the voltage source as a function of incident power was determined, which is equal to $|1-T|{ }^{44}$ The $E_{x}$ power reradiated in the forward direction was determined by simulating the power dissipated by the resistor, $R_{\mathrm{Fwd}}$, as a function of incident power. The resultant $\left|E_{y}\right|$ and $\left|E_{x}\right|$ terms emitted through the sample as a function of frequency and backgate voltage are shown in Figure 4(b) and compared with the equivalent Comsol simulation values reported in Figure 4(a). The two waveforms obtained from Comsol simulations and the equivalent model are in very good agreement, thus reinforcing the validity of the circuital analysis. Both simulations have a bonding resonance at $1.65 \mathrm{THz}$, as characterized by a minimum in transmitted $\left|E_{y}\right|$
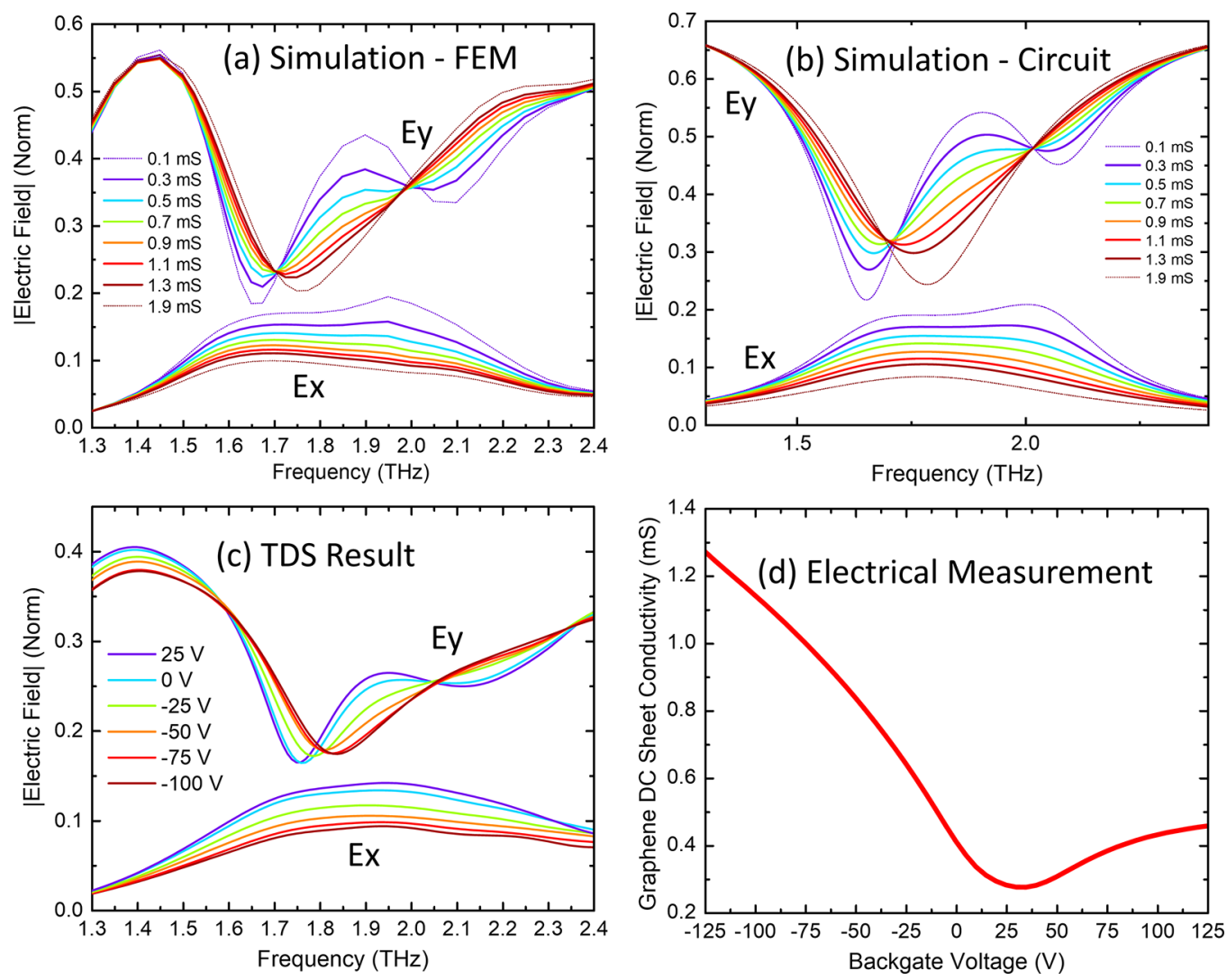

Figure 4. Transmitted $\left|E_{y}\right|$ and $\left|E_{x}\right|$ components as a function of graphene conductivity and frequency determined using (a) finite element method simulation and (b) equivalent circuit model simulation. (c) TDS measurement of transmitted $\left|E_{y}\right|$ and $\left|E_{x}\right|$ components as a function of backgate voltage. (d) Electrical measurement of graphene DC real conductivity as a function of backgate voltage. 

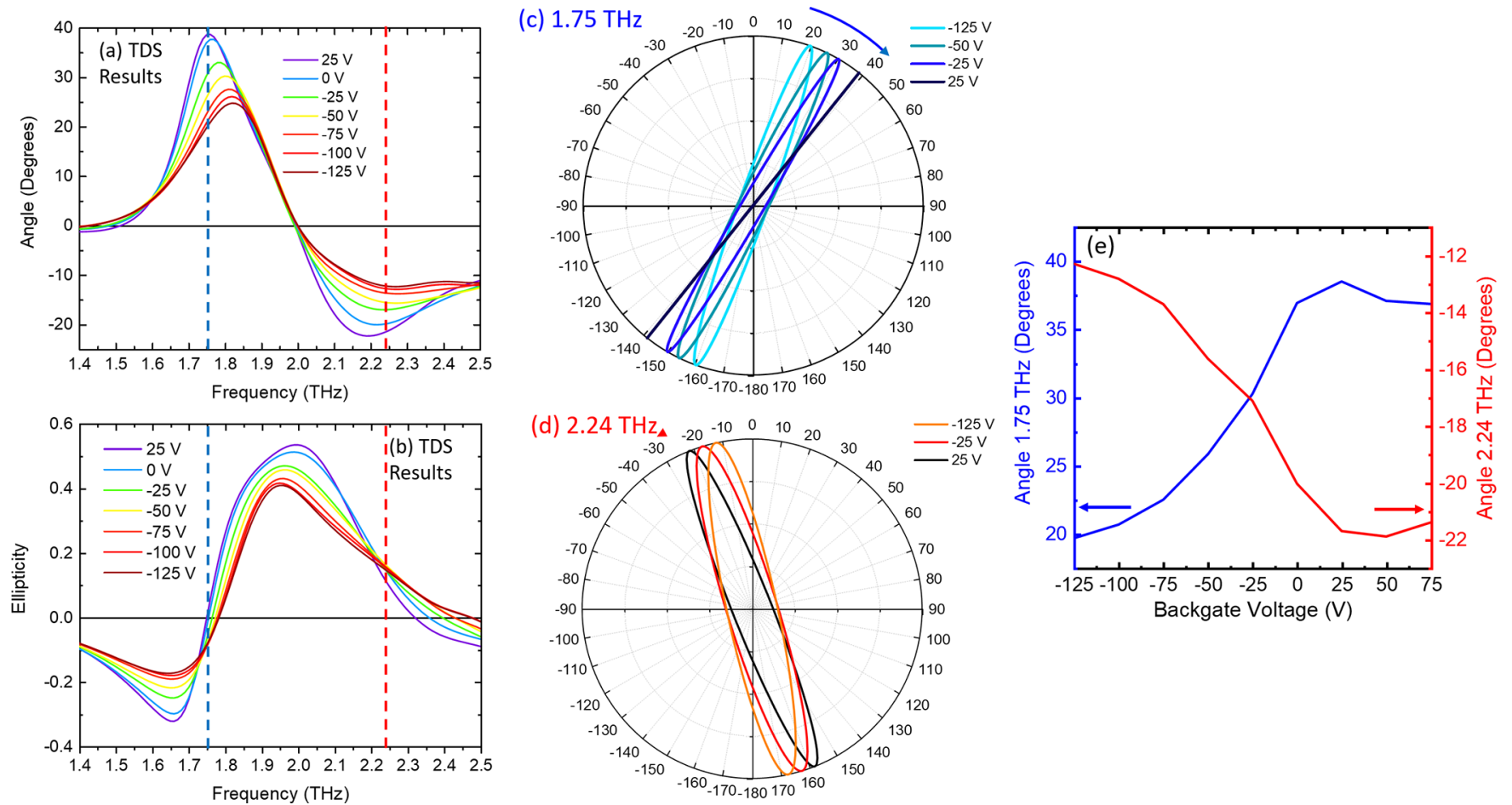

Figure 5. (a) TDS results of polarization angle of emitted radiation through the device as a function of frequency and backgate voltage. (b) Corresponding ellipticity of radiation through the device. (c and d) Polar plots of radiation measured after the device at $1.75 \mathrm{and} 2.24 \mathrm{THz}$, respectively, for different backgate voltages. (e) Polarization angle as a function of backgate voltage at 1.75 and $2.24 \mathrm{THz}$.

radiation, and have a broad peak in transmitted $\left|E_{x}\right|$ radiation. There is a weaker dip in $\left|E_{y}\right|$ at $2.07 \mathrm{THz}$, indicating the antibonding resonance. The lithographic tuning of the bright and dark resonators was chosen to create this lopsided coupled resonance condition, as described in ref 44 , to demonstrate a larger polarization modulation depth at $1.65 \mathrm{THz}$. As the graphene conductivity increases, the coupled resonator regime is converted into a single-resonator regime, with an $\left|E_{y}\right|$ transmission dip at $1.8 \mathrm{THz}$ and a reduction in the overall value of $\left|E_{x}\right|$. Accordingly, the polarization plane angle of transmitted radiation, with a frequency around the bonding or antibonding resonance, is rotated as the graphene conductivity is changed.

Measurements: Broadband THz Time Domain Spectroscopy. The broadband $\mathrm{THz}$ transmission response of the fabricated device was measured in a polarization-sensitive time domain spectroscopy (TDS) system from Menlo Systems, model $\mathrm{k} 15$, operated in transmission mode as described in Section SI.4. $\left|E_{y}(f)\right|$ and $\left|E_{x}(f)\right|$ as a function of backgate voltage were measured in the TDS setup, normalized to $\left|E_{y}(f)\right|$ measured with no sample present, with the results shown in Figure 4(c). Electrical characterization of the large graphene area fabricated on chip was used to obtain the graphene conductivity at different backgate voltages. Two Keithly 2400 source-drain units were used, with one supplying $5 \mu \mathrm{A}$ of current across the source and drain while measuring the required voltage and the other used to apply a variable DC voltage across the backgate. The resultant graphene DC conductivity as a function of backgate voltage is shown in Figure 4(d) with the Dirac point visible at around $+30 \mathrm{~V}$.

The overall emitted $\left|E_{x}(f)\right|$ amplitude is maximum when the backgate voltage is set to $+25 \mathrm{~V}$, in very good agreement with the Dirac point retrieved from the large graphene area. The I $E_{x}(f) \mid$ amplitude reduces as the backgate voltage, $V_{\mathrm{BG}}$, is lowered from the Dirac point due to the increased conductivity of the graphene, which is shunting the capacitive gap, dissipating more power, and dampening the resonance. The coupled resonator bonding and antibonding resonances are clearly visible at $+25 \mathrm{~V}$, with $\left|E_{y}\right|$ transmission dips at 1.75 and $2.15 \mathrm{THz}$, respectively. For $V_{\mathrm{BG}}=-100 \mathrm{~V}$, there is only one visible resonance given by a transmission dip around 1.85 THz. Therefore, at the fixed frequency of $1.72 \mathrm{THz},\left|E_{y}\right|$ increases by approximately $60 \%$ while $E_{x}$ reduces by approximately $40 \%$, leading to a change in the $\left|E_{y}\right| /\left|E_{x}\right|$ ratio from 1.2 to 2.3 , which corresponds to a change in polarization angle of around $20^{\circ}$. There is very good qualitative agreement between the measured E-field components and the simulation curves obtained with the FEM simulation and the theoretical circuit model shown in Figure 4(a) and (b), respectively. The resonance frequencies of the TDS results are blue-shifted by around $75 \mathrm{GHz}$ when compared to simulation, due to the discrepancy in material permittivity values in the simulation when compared to the TDS results. For the FEM simulation, there is a larger tuning range for $\left|E_{y}\right|$ and $\left|E_{x}\right|$, with a change in the $\left|E_{y}\right| /\left|E_{x}\right|$ ratio from 1.1 to 3 shown in Figure 4(a), using conductivities of 0.1 and $1.9 \mathrm{mS}$ shown by the dotted curves. These values are beyond the achievable range for the device, with the tuning range measured to be between 0.3 and $1.3 \mathrm{mS}$. The larger simulation range was implemented to gain insight into the potential tuning capabilities for the device if graphene with a different conductivity range was used. By implementing the measured graphene conductivity tuning range into the FEM simulation, the ratio $\left|E_{y}\right| /\left|E_{x}\right|$ is modulated between 1.2 and 2.5 , thus yielding a remarkable agreement (within 10\%) with the measurements acquired using the THz-TDS.

In order to fully analyze the results of the polarization tuning, the relative phase between the $E_{y}$ and $E_{x}$ terms must be considered along with the relative magnitudes. The angle and 


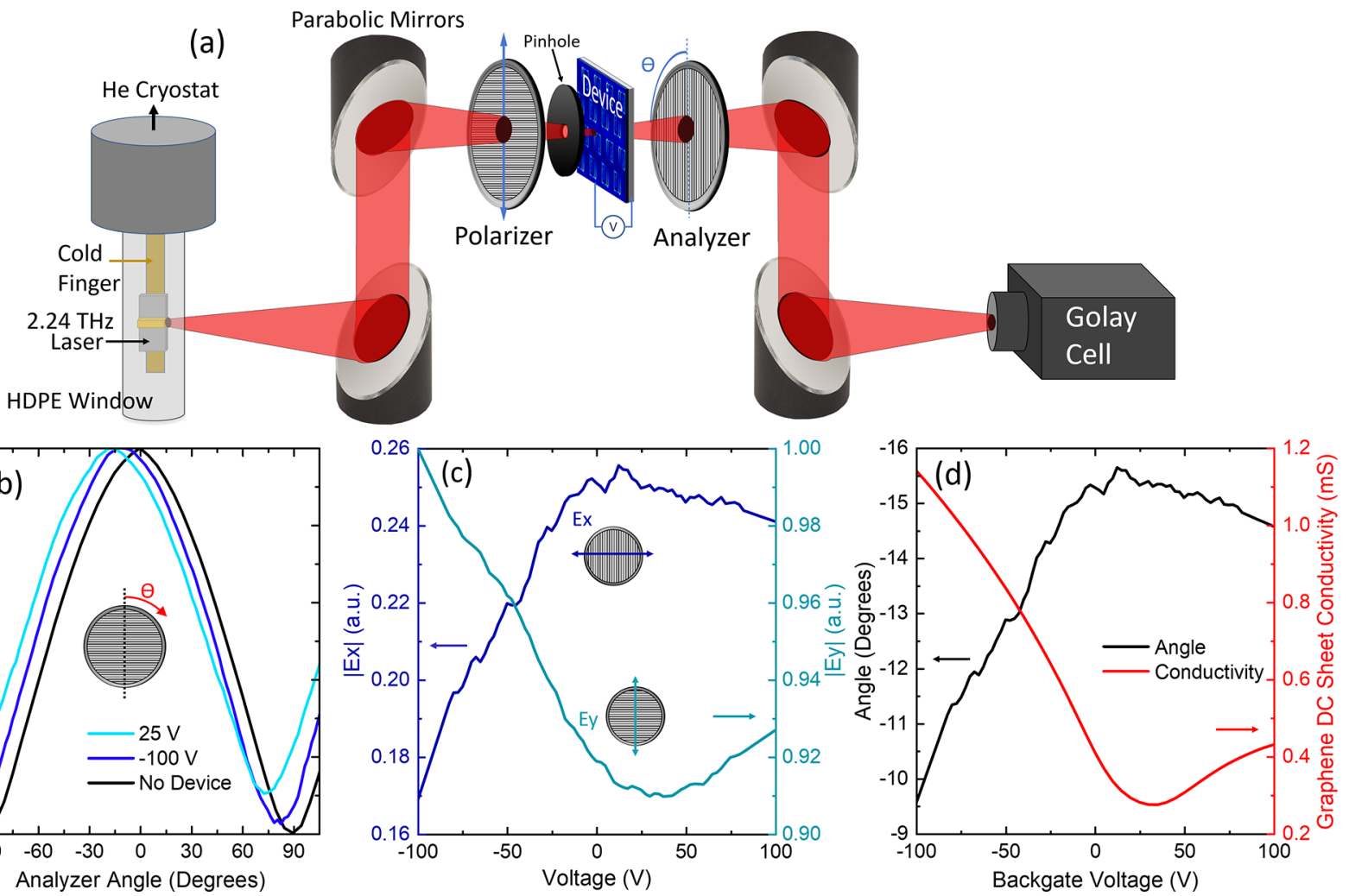

Figure 6. (a) Illustration of QCL polarization modulation setup. (b) Electric field magnitude transmitted through the device, measured by a Golay cell as a function of analyzer angle. (c) Amplitude of transmitted electric field in the $E_{x}$ and $E_{y}$ directions as a function of backgate voltage. (d) Resultant polarization angle through the device as a function of backgate voltage, plotted alongside the electrical measurement of graphene conductivity.

ellipticity of the transmitted radiation can be determined from these two components using the relations described in Section SI.5. The transmitted polarization condition can be characterized generally as elliptical polarization, with an ellipticity value ranging from 0 (perfectly linear polarization) to \pm 1 (circular-polarized radiation). For many applications where a polarization modulation is required, ideally the ellipticity would remain as close to 0 as possible as the plane polarization angle is rotated. Plots of the resultant transmitted polarization angle and ellipticity are shown in Figure 5(a) and (b), respectively.

The measured rotation angle presents positive values at low frequencies, peaking at around $40^{\circ}$ as the frequency reaches $1.75 \mathrm{THz}$, with the backgate voltage set to $+25 \mathrm{~V}$. At this frequency, the corresponding ellipticity is close to 0 , since the $E_{x}$ and $E_{y}$ components are almost in phase. As the frequency is increased beyond this point, the polarization angle reduces until it crosses zero at $2.0 \mathrm{THz}$. In this case the polarization is highly elliptical, with the $E_{y}$ and $E_{x}$ components being out of phase by around $\pi / 2$. As the frequency is increased further, the polarization angle becomes negative, peaking at $2.2 \mathrm{THz}$, corresponding to a phase difference of $\pi$, representing completely linear polarization once again. According to Figure $5(\mathrm{a})$, the maximum modulation of the polarization plane angle is achieved at $1.75 \mathrm{THz}$ with the polarization angle increasing from $20^{\circ}$ to $40^{\circ}$ and the ellipticity magnitude remaining below 0.1 as the backgate voltage is decreased from $+25 \mathrm{~V}$ to $-125 \mathrm{~V}$. The polarization at $2.24 \mathrm{THz}$ is modulated from $-13^{\circ}$ to $-22^{\circ}$, with the ellipticity remaining stable at around 0.15 . Polar plots illustrating the polarization condition at these two frequencies are shown in Figure 5(c) and (d), and the resultant polarization angles as a function of the backgate voltage are plotted in Figure 5(e). The polarization angle trends match the graphene conductivity measurements shown in Figure 4(d), with the angle magnitude increasing in a nearly linear fashion between -75 and $0 \mathrm{~V}$, before reaching a maximum at the Dirac point around $+30 \mathrm{~V}$, and then decreasing in magnitude as the voltage is increased beyond this point. The polarization modulation in the $2.24 \mathrm{THz}$ frequency region, although presenting a reduced modulation depth because of the aforementioned lopsided lithographic mismatch, intercepts the spectral range where terahertz QCLs emit strongly. This frequency overlap allowed the integration of this polarization modulator device with a $\mathrm{THz}$ QCL, thus paving the way for its implementation in polarization spectroscopy or for the fundamental investigation of topological insulator materials to aid the development of novel spintronic devices. ${ }^{46}$

QCL Polarization Control. The polarization modulator was integrated with a $\mathrm{THz}$ QCL fabricated into a single plasmon waveguide from a bound-to-continuum design and emitting in single-mode frequency throughout the dynamic range at $2.24 \mathrm{THz}$. The frequency spectrum for this laser is shown in Section SI.6. The experimental setup used to tune the polarization of QCL emission is schematically reported in Figure 6(a).

The QCL was mounted on a liquid-He flow cryostat with the emitted radiation collimated using a $7 \mathrm{~cm}$ long focal length parabolic mirror. A second mirror with a focal length of $5 \mathrm{~cm}$ was inserted to focus the radiation onto the metamaterial polarization device. Two $10 \mathrm{~cm}$ long focal length parabolic mirrors were then used to collect the transmitted output and focus the radiation onto a Tydex Golay cell for detection. Two 
grid wire polarizers, identified here as the polarizer and analyzer, were inserted before and after the sample, to allow for precise control of the incoming radiation and to probe the transmitted E-field polarization component. The analyzer was mounted onto an active rotational stage to probe the electric field emitted by the device at various polarization angles. Typically the QCL was operated with a $10 \mathrm{kHz}$ repetition rate with a duty cycle of $15 \%$, and a further $5 \mathrm{~Hz}$ gate frequency was applied for lock-in detection of the Golay-measured power.

The normalized electric field as a function of the analyzer angle is shown by the black curve in Figure 6(b) taken before inserting the sample. The same operation was performed with the device in the optical path for backgate voltages of $+25 \mathrm{~V}$ (Dirac point) and $-100 \mathrm{~V}$, as shown in Figure 6(b). The rotation angle changes from about $-9^{\circ}$ to $-16^{\circ}$ when the backgate voltage is swept from $-100 \mathrm{~V}$ to $+25 \mathrm{~V}$, respectively, thus yielding a $7^{\circ}$ modulation range, in good agreement with the THz-TDS measurements at $2.24 \mathrm{THz}$. Similar measurements were performed by setting the analyzer to $0^{\circ}$ and $90^{\circ}$ to measure the emitted $\left|E_{y}\right|$ and $\left|E_{x}\right|$ terms, respectively, as a function of backgate voltage. These results are reported in Figure $6(\mathrm{c})$, with the corresponding plane polarization angle plotted in Figure 6(d) alongside the graphene conductivity measurements, reporting a consistent trend. When the graphene conductivity is minimum, the polarization angle is maximum to within $\pm 10 \mathrm{~V}$, commensurate to Dirac point variation of exposed graphene. Further to this, the polarization angle trend follows an inverse relation with respect to the conductivity, with high conductivities corresponding to low angle rotations, as predicted in Figure 5. The modulation speed of the QCL polarization was also tested, with the modulation depth as a function of modulation frequency shown in Section SI.7. Modulation speeds of up to $5 \pm 1 \mathrm{MHz}$ are demonstrated for this device; however, modifications to the device size and substrate resistivity could increase this modulation speed to $>100 \mathrm{MHz}$, as demonstrated in ref 36 .

\section{CONCLUSION}

Active polarization modulation has been demonstrated using a 2D chiral metamaterial device integrated with electrically tunable graphene. The device was fully characterized with a THz-TDS spectrometer, yielding remarkable agreement with the finite element simulations and equivalent circuit model predictions. A continuous polarization angle tuning of $20^{\circ}$ was achieved at $1.75 \mathrm{THz}$ by tuning the applied backgate voltage. This device has also been successfully integrated with a $\mathrm{THz}$ QCL operating at $2.24 \mathrm{THz}$, demonstrating active polarization modulation of $7^{\circ}$ with modulation speeds of up to $5 \pm 1 \mathrm{MHz}$. The integrated polarization modulation of QCL emission opens new scenarios for the all-electrical fast control of $\mathrm{THz}$ radiation. This device represents an important demonstration of how standard $\mathrm{THz}$ sources can be actively polarization controlled at electronic speeds, for a plethora of applications in $\mathrm{THz}$ polarization spectroscopy, real time material characterization, and $\mathrm{THz}$ communication systems of the future.

\section{ASSOCIATED CONTENT}

\section{S Supporting Information}

The Supporting Information is available free of charge on the ACS Publications website at DOI: 10.1021/acsphotonics.9b00411.
Lithographic tuning parameters for the device design, the frequency-dependent material parameters used in the Comsol simulation, the parameters used in the equivalent circuit model, TDS polarization setup, including the polarization angle and ellipticity mathematical definitions, a section of the QCL spectrum of the laser, and measurement of the modulation speed of the polarization device coupled with the QCL (PDF)

\section{AUTHOR INFORMATION}

\section{Corresponding Author}

*E-mail: sjk80@cam.ac.uk.

\section{ORCID}

Stephen J. Kindness: 0000-0002-0776-5896

Philipp Braeuninger-Weimer: 0000-0001-8677-1647

Riccardo Degl'Innocenti: 0000-0003-2655-1997

\section{Funding}

S.J.K. acknowledges the Integrated Photonic and Electronic Systems CDT (Grant No. EP/L015455/1) for funding and support. S.J.K., N.W.A., B.W., W.M., H.E.B., D.A.R., and R.D. acknowledge financial support from the Engineering and Physical Sciences Research Council (EPSRC) (Grant No. EP/ P021859/1, Hyper Terahertz). W.M. thanks the George and Lillian Schiff Foundation of the University of Cambridge for financial support and is grateful for the Honorary ViceChancellor's Award of the Cambridge Trust. R.D. acknowledges support from the EPSRC (Grant No EP/S019383/1) and from the Royal Society (RSG/R1/180148 - Research Grant). S.H. and P.B.W. acknowledge funding from the EPSRC (Grant No. EP/K016636/1). P.B.W. acknowledges the EPSRC Cambridge NanoDTC (Grant No. EP/G037221/1). K.D. acknowledges financial support from the EPSRC (Grant No. EP/S019324/1, MQIC).

\section{Notes}

The authors declare no competing financial interest.

\section{REFERENCES}

(1) Benedetto, S.; Member, S.; Poggiolini, P. Theory of Polarization Shift Keying Modulation. IEEE Trans. Commun. 1992, 40, 708.

(2) Oshima, N.; Hashimoto, K.; Suzuki, S.; Asada, M. Terahertz Wireless Data Transmission With Frequency and Polarization Division Multiplexing Using Resonant-Tunneling-Diode Oscillators. IEEE Trans. Terahertz Sci. Technol. 2017, 7, 593-598.

(3) Jenkins, G. S.; Sushkov, A. B.; Schmadel, D. C.; Butch, N. P.; Syers, P.; Paglione, J.; Drew, H. D. Terahertz Kerr and reflectivity measurements on the topological insulator Bi2Se3. Phys. Rev. B: Condens. Matter Mater. Phys. 2010, 82, DOI: 10.1103/PhysRevB.82.125120.

(4) Plank, H.; Ganichev, S. D. A review on terahertz photogalvanic spectroscopy of Bi2Te3- and Sb2Te3-based three dimensional topological insulators. Solid-State Electron. 2018, 147, 44-50.

(5) Zhang, L.; Zhong, H.; Deng, C.; Zhang, C.; Zhao, Y. Characterization of birefringent material using polarization-controlled terahertz spectroscopy. Opt. Express 2010, 18, 20491.

(6) Markl, D.; Bawuah, P.; Ridgway, C.; van den Ban, S.; Goodwin, D. J.; Ketolainen, J.; Gane, P.; Peiponen, K.-E.; Zeitler, J. A. Fast and non-destructive pore structure analysis using terahertz time-domain spectroscopy. Int. J. Pharm. 2018, 537, 102-110.

(7) Markl, D.; Strobel, A.; Schlossnikl, R.; Bøtker, J.; Bawuah, P.; Ridgway, C.; Rantanen, J.; Rades, T.; Gane, P.; Peiponen, K.-E.; Zeitler, J. A. Characterisation of pore structures of pharmaceutical tablets: A review. Int. J. Pharm. 2018, 538, 188-214. 
(8) Xu, J.; Ramian, G.; Galan, J. Terahertz circular dichroism spectroscopy: A potential approach to the in situ detection of life's metabolic and genetic machinery. Astrobiology 2003, 3, 489.

(9) Xu, J.; Galan, J.; Ramian, G.; Savvidis, P.; Scopatz, A.; Birge, R. R.; Allen, S. J.; Plaxco, K. Terahertz circular dichroism spectroscopy of biomolecules. Proc. SPIE 2003, 19.

(10) Hirota, Y.; Hattori, R.; Tani, M.; Hangyo, M. Polarization modulation of terahertz electromagnetic radiation by four-contact photoconductive antenna; Opt. Express 2006, 14, 4486.

(11) Hibberd, M. T.; Lake, D. S.; Johansson, N. A.; Thomson, T.; Jamison, S. P.; Graham, D. M. Magnetic-field tailoring of the terahertz polarization emitted from a spintronic source. Appl. Phys. Lett. 2019, 114, 031101.

(12) Vitiello, M. S.; Scalari, G.; Williams, B.; De Natale, P. Quantum cascade lasers: 20 years of challenges. Opt. Express 2015, 23, 5167.

(13) Liu, X.; Chen, X.; Parrott, E. P. J.; Pickwell-MacPherson, E. Exploiting a metal wire grating in total internal reflection geometry to achieve achromatic polarization conversion. Photonics Res. 2017, 5 , 299.

(14) Liu, X.; Chen, X.; Parrott, E. P. J.; Han, C.; Humbert, G.; Crunteanu, A.; Pickwell-MacPherson, E. Invited Article: An active terahertz polarization converter employing vanadium dioxide and a metal wire grating in total internal reflection geometry. APL Photonics 2018, 3, 051604.

(15) Fan, R.-H.; Zhou, Y.; Ren, X.-P.; Peng, R.-W.; Jiang, S.-C.; Xu, D.-H.; Xiong, X.; Huang, X.-R.; Wang, M. Freely Tunable Broadband Polarization Rotator for Terahertz Waves. Adv. Mater. 2015, 27, 1201-1206.

(16) Yamada, I.; Takano, K.; Hangyo, M.; Saito, M.; Watanabe, W. Terahertz wire-grid polarizers with micrometer-pitch $\mathrm{Al}$ gratings. Opt. Lett. 2009, 34, 274.

(17) Woo, J. M.; Hussain, S.; Jang, J.-H. A terahertz in-line polarization converter based on through-via connected double layer slot structures. Sci. Rep. 2017, 7, 42952.

(18) Parvinnezhad Hokmabadi, M.; Wilbert, D. S.; Kung, P.; Kim, S. M. Polarization-Dependent, Frequency-Selective THz Stereometamaterial Perfect Absorber. Phys. Rev. Appl. 2014, 1, 044003.

(19) Chen, H. T.; Taylor, A. J.; Yu, N. A review of metasurfaces: Physics and applications. Rep. Prog. Phys. 2016, 79, 076401.

(20) Grady, N. K.; Heyes, J. E.; Chowdhury, D. R.; Zeng, Y.; Reiten, M. T.; Azad, A. K.; Taylor, A. J.; Dalvit, D. A. R.; Chen, H.-T. Terahertz Metamaterials for Linear Polarization Conversion and Anomalous Refraction. Science 2013, 340, 1304-1307.

(21) Fang, F.; Cheng, Y.; Liao, H. Giant optical activity and circular dichroism in the terahertz region based on bi-layer Y-shaped chiral metamaterial. Optik 2014, 125, 6067-6070.

(22) Wu, L.; Yang, Z.; Cheng, Y.; Lu, Z.; Zhang, P.; Zhao, M.; Gong, R.; Yuan, X.; Zheng, Y.; Duan, J. Electromagnetic manifestation of chirality in layer-by-layer chiral metamaterials. Opt. Express 2013, 21, 5239.

(23) Kan, T.; Isozaki, A.; Kanda, N.; Nemoto, N.; Konishi, K.; Takahashi, H.; Kuwata-Gonokami, M.; Matsumoto, K.; Shimoyama, I. Enantiomeric switching of chiral metamaterial for terahertz polarization modulation employing vertically deformable MEMS spirals. Nat. Commun. 2015, 6, 8422.

(24) Zhang, S.; Zhou, J.; Park, Y.-S.; Rho, J.; Singh, R.; Nam, S.; Azad, A. K.; Chen, H.-T.; Yin, X.; Taylor, A. J.; Zhang, X. Photoinduced handedness switching in terahertz chiral metamolecules. Nat. Commun. 2012, 3, 942.

(25) Cong, L.; Srivastava, Y. K.; Zhang, H.; Zhang, X.; Han, J.; Singh, R. All-optical active $\mathrm{THz}$ metasurfaces for ultrafast polarization switching and dynamic beam splitting. Light: Sci. Appl. 2018, 7, 28.

(26) Zhou, J.; Chowdhury, D. R.; Zhao, R.; Azad, A. K.; Chen, H. T.; Soukoulis, C. M.; Taylor, A. J.; O’Hara, J. F. Terahertz chiral metamaterials with giant and dynamically tunable optical activity. Phys. Rev. B: Condens. Matter Mater. Phys. 2012, 86, 035448.

(27) Sensale-Rodriguez, B.; Yan, R.; Kelly, M. M.; Fang, T.; Tahy, K.; Hwang, W. S.; Jena, D.; Liu, L.; Xing, H. G. Broadband graphene terahertz modulators enabled by intraband transitions. Nat. Commun. 2012, 3, 780.

(28) Degl'Innocenti, R.; Kindness, S. J.; Beere, H. E.; Ritchie, D. A. All-integrated terahertz modulators. Nanophotonics 2018, 7, 127-144.

(29) Nashima, S.; Morikawa, O.; Takata, K.; Hangyo, M. Measurement of optical properties of highly doped silicon by terahertz time domain reflection spectroscopy. Appl. Phys. Lett. 2001, 79, 3923-3925.

(30) Yao, Y.; Kats, M. A.; Shankar, R.; Song, Y.; Kong, J.; Loncar, M.; Capasso, F. Wide wavelength tuning of optical antennas on graphene with nanosecond response time. Nano Lett. 2014, 14, 214219.

(31) Yao, Y.; Kats, M. A.; Genevet, P.; Yu, N.; Song, Y.; Kong, J.; Capasso, F. Broad electrical tuning of graphene-loaded plasmonic antennas. Nano Lett. 2013, 13, 1257-1264.

(32) Degl'Innocenti, R.; Jessop, D. S.; Shah, Y. D.; Sibik, J.; Zeitler, J. A.; Kidambi, P. R.; Hofmann, S.; Beere, H. E.; Ritchie, D. A. Low-Bias Terahertz Amplitude Modulator Based on Split-Ring Resonators and Graphene. ACS Nano 2014, 8, 2548-2554.

(33) Wei, B.; Kindness, S.; Almond, N.; Wallis, R.; Wu, Y.; Ren, Y.; Shi, S.; Braeuninger-Weimer, P.; Hofmann, S.; Beere, H.; Ritchie, D.; Degl'Innocenti, R. Amplitude stabilization and active control of a terahertz quantum cascade laser with a graphene loaded split-ringresonator array. Appl. Phys. Lett. 2018, 112, 201102.

(34) Degl'Innocenti, R.; Jessop, D. S.; Sol, C. W.; Xiao, L.; Kindness, S. J.; Lin, H.; Zeitler, J. A.; Braeuninger-Weimer, P.; Hofmann, S.; Ren, Y.; Kamboj, V. S.; Griffiths, J. P.; Beere, H. E.; Ritchie, D. A. Fast Modulation of Terahertz Quantum Cascade Lasers Using Graphene Loaded Plasmonic Antennas. ACS Photonics 2016, 3, 464-470.

(35) Kindness, S. J.; Jessop, D. S.; Wei, B.; Wallis, R.; Kamboj, V. S.; Xiao, L.; Ren, Y.; Braeuninger-Weimer, P.; Aria, A. I.; Hofmann, S.; Beere, H. E.; Ritchie, D. A.; Degl'Innocenti, R. External amplitude and frequency modulation of a terahertz quantum cascade laser using metamaterial/graphene devices. Sci. Rep. 2017, 7, 1-10.

(36) Jessop, D. S.; Kindness, S. J.; Xiao, L.; Braeuninger-Weimer, P.; Lin, H.; Ren, Y.; Ren, C. X.; Hofmann, S.; Zeitler, J. A.; Beere, H. E.; Ritchie, D. A.; Degl'Innocenti, R. Graphene based plasmonic terahertz amplitude modulator operating above $100 \mathrm{MHz}$. Appl. Phys. Lett. 2016, 108.171101

(37) Radziunas, M.; Wünsche, H.-J.; Krauskopf, B.; Wolfrum, M. External cavity modes in Lang-Kobayashi and traveling wave models. Proc. SPIE 2006, 6184, 61840X-61840X-9.

(38) Singh, R.; Rockstuhl, C.; Lederer, F.; Zhang, W. Coupling between a dark and a bright eigenmode in a terahertz metamaterial. Phys. Rev. B: Condens. Matter Mater. Phys. 2009, 79, 085111.

(39) Cao, W.; Singh, R.; Zhang, C.; Han, J.; Tonouchi, M.; Zhang, W. Plasmon-induced transparency in metamaterials: Active near field coupling between bright superconducting and dark metallic mode resonators. Appl. Phys. Lett. 2013, 103, 101106.

(40) Chen, J.; Wang, P.; Chen, C.; Lu, Y.; Ming, H.; Zhan, Q. Plasmonic EIT-like switching in bright-dark-bright plasmon resonators. Opt. Express 2011, 19, 5970.

(41) Zhang, S.; Genov, D. A.; Wang, Y.; Liu, M.; Zhang, X. PlasmonInduced Transparency in Metamaterials. Phys. Rev. Lett. 2008, 101, 047401 .

(42) Hofmann, S.; Braeuninger-Weimer, P.; Weatherup, R. S. CVDenabled graphene manufacture and technology. J. Phys. Chem. Lett. 2015, 6, 2714-2721.

(43) Braeuninger-Weimer, P.; Brennan, B.; Pollard, A. J.; Hofmann, $\mathrm{S}$. Understanding and Controlling $\mathrm{Cu}$-Catalyzed Graphene Nucleation: The Role of Impurities, Roughness, and Oxygen Scavenging. Chem. Mater. 2016, 28, 8905-8915.

(44) Kindness, S. J.; Almond, N. W.; Wei, B.; Wallis, R.; Michailow, W.; Kamboj, V. S.; Braeuninger-Weimer, P.; Hofmann, S.; Beere, H. E.; Ritchie, D. A.; Degl'Innocenti, R. Active Control of Electromagnetically Induced Transparency in a Terahertz Metamaterial Array with Graphene for Continuous Resonance Frequency Tuning. Adv. Opt. Mater. 2018, 6, 1800570. 
(45) Zou, Y.; Tassin, P.; Koschny, T.; Soukoulis, C. M. Interaction between graphene and metamaterials: split rings vs wire pairs. Opt. Express 2012, 20, 12198.

(46) McIver, J. W.; Hsieh, D.; Steinberg, H.; Jarillo-Herrero, P.; Gedik, N. Control over topological insulator photocurrents with light polarization. Nat. Nanotechnol. 2012, 7, 96-100. 